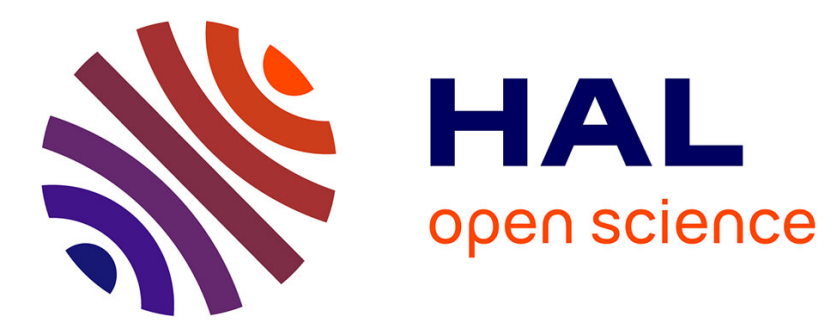

\title{
Sociologie du mariage en classe de 3e: lectures et écritures
}

Jean-Marie Privat

\section{To cite this version:}

Jean-Marie Privat. Sociologie du mariage en classe de 3e: lectures et écritures. Pratiques: linguistique, littérature, didactique, 1986, 50, pp.79-100. 10.3406/prati.1986.1389 . hal-03180241

\section{HAL Id: hal-03180241 \\ https://hal.univ-lorraine.fr/hal-03180241}

Submitted on 24 Mar 2021

HAL is a multi-disciplinary open access archive for the deposit and dissemination of scientific research documents, whether they are published or not. The documents may come from teaching and research institutions in France or abroad, or from public or private research centers.
L'archive ouverte pluridisciplinaire HAL, est destinée au dépôt et à la diffusion de documents scientifiques de niveau recherche, publiés ou non, émanant des établissements d'enseignement et de recherche français ou étrangers, des laboratoires publics ou privés. 


\section{Sociologie du mariage en classe de $3 e$ : lectures et écritures} Jean-Marie Privat

\section{Citer ce document / Cite this document :}

Privat Jean-Marie. Sociologie du mariage en classe de $3 e$ : lectures et écritures. In: Pratiques : linguistique, littérature, didactique, $\mathrm{n}^{\circ} 50,1986$. Les paralittératures. pp. 79-100;

doi : https://doi.org/10.3406/prati.1986.1389

https://www.persee.fr/doc/prati_0338-2389_1986_num_50_1_1389

Fichier pdf généré le 13/07/2018 


\section{SOCIOLOGIE DU MARIAGE EN CLASSE DE $3^{\mathrm{e}}$ : LECTURES ET ÉCRITURES}

Jean-Marie PRIVAT

Pour lutter contre la gratuité apparente des lectures scolaires - qui engendrent souvent, parmi les élèves issus des classes populaires, le plus grand désintérêt - le recours initial aux écrits utilitaires est, précisément, fort utile!

Nous nous proposons en effet de montrer comment, à partir de la lecture du très administratif "Bulletin officiel de la ville de Lyon "), une classe de $3^{\mathrm{e}}$ (ou au-delà) peut (se) construire elle-même un réel savoir critique sur un aspect du fonctionnement objectif d'une société et de sa culture, et activer, dans la logique même d'une interrogation vivante, ses compétences de lecturesécritures (1).

Les hasards(?) d'une conversation anecdotique ( $(M$., votre femme est prof aussi... ? >) qui couvre un questionnement existentiel implicite vont amener la classe à s'interroger d'une manière systématique sur une problématique dont chacun s'accorde à admettre, rapidement, l'intérêt théorique... et personnel: comment s'organisent les relations institutionnelles a l'autre (sexe), comment se (re-)produisent les rapports sociaux dans le cadre du mariage, autrement dit "qui épouse qui?».

Ces questions posées, vécues passionnellement, seront abordées par le biais d'un document actuel mais objectif, " froid»), qui ménage ainsi les sensibilités adolescentes, tout en jouant d'un incontestable effet de surprise.

Un étonnement résigné - encore une lubie de prof ? - saisit en effet mes nouveaux élèves lorsque je distribue la photocopie (cf. infra, l'extrait reproduit) des pages de I'ÉTAT CIVIL concernant les "Promesses de mariage dans les mairies d'arrondissements pour y être affichées, du 12 au 20 août 1983 inclus $).$

(1) Ce travail a été conduit dans un collège des Minguettes, au cours de l'année 1983-1984. Nous ne nous interdirons pas cependant d'utiliser ou de citer des expériences et travaux plus récents. 


\section{ÉTAT CIVIL}

Promesses de mariage déposées dans les mairies d'arrondissements pour y être affichées, du 12 au 20 aoút 1983 inclus.

\section{PREMIER ARRONDISSEMENT}

Turbelin Olivier, conducteur de trav., r. du Bât-d'Argent, 1. Lerouge Michèle, agent commercial, $r$. du Bât-d'Argent, 1.

Levera Gerard, inspecteur princ. aux P.T.T., r. Faidherbe, 18, Sathonay-Camp (Rhône). Dor Christine, infirmière, r. Diderot, 9.

Carras Michel, agent de méthode, mtée des Carmélites, 30.

Boulard Dominique, dactylocodeuse, ch. de la Caille, 1, Vaulx-en-Velin (Rhône).

Raquin Christian, infirmier, "Valency»), Pollionnay (Rhône).

Michallet Béatrix, infirmière, r. de l'Alma, 10.

\section{DEUXIĖME ARRONDISSEMENT}

Rivoire Bruno, empl. de régie, r. Victor-Hugo, 7.

Fontbonne Elisabeth, manipulatrice en électro-radiologie, r. Victor-Hugo, 7.

Roy de Lachaise Christophe, attaché de direction commercial, pl. Carnot, 6.

Michon du Marais Marguerite, étudiante, r. du Planit, 1, Sainte-Foy-lès-Lyon (Rhône).

Saighi Djony-Faouzi, surveillant pénitentiaire, qu. Perrache, 12. Febvre Nadine, ouvrière, r. de la Batavarde, 6, Perrigny (Jura).

Rousson François, enseignant, r. Victor-Hugo, 35.

Orsini Marilyn, enseignante, r. d'Arsonval, 18, Château-Arnoux (Alpes de Haute-Provence).

Pesle Hervé, Ingénieur commercial, r. des Remparts-d'Alnay, 15. Dumont Isabelle, s.p., La Ferté-Milon (Aisne).

Alcantara Michel, étudiant, r. Ludovic-Arrachart, 5. Dupuis Isabelle, étudiante, r. Sala, 9.

Atuyer Richard, réviseur comptable, r. Victor-Hugo, 40. Ranel Sylvie, s.p., Le Bourg, Lentilly (Rhône).

Oustric Thierry, agent E.D.F., r. de la Barre, 5. Rigot Joëlle, assistante comptable, $r$. de la Barre, 5.

Cotin Didier, boulanger, r. Casimir-Périer, 18. Blanc Corinne, fleuriste, av. Félix-Faure, 172.

Vincent Dominique, cuisinier, Poleymieux-au-Mont-d'Or (Rhône). Hernandez Marie, opératrice de saisie, crs Bayard, 22.

\section{TROISIEME ARRONDISSEMENT}

Antonini Maxim, cadre commercial, r. Jeanne-d'Arc, 49 bis. Maréchal Françoise, archit. visagiste, $r$. des Montées, 16, Orléans (Loiret).

Rivat Alain, plâtrier-peintre, av. Lacassagne, 126. Kozaoustopoulos Michèle, s.p., av. Lacassagne, 126.

Lesueur Christophe, étudiant architecture, r. Gabriel-Fauré, 6, Chassieu (Rhône). Roch Valérie, attachée de direction, $r$. Duguesclin, 181.

Picollet Pierre, agent technique, crs Eugénie, 38. Baudonnet Claire, laborantine, r. de la Poterie, Loriol-sur-Drôme (Drôme).

Deneux François, ingénieur, av. Félix-Faure, 196. Schleicher Régine, rédactrice, r. Sainte-Hélène, 2.

Rety Georges, agent de sécurité, av. Lacassagne, 143. Tanton Patricia, mécanicienne fourrure, av. Lacassagne, 143.

Andreu Bernard, mécanicien, $r$. Jeanne-Hachette, 1.

Guerle Michèle, s.p., ch. du Gerbier, 4, Vaulx-en-V. (Rh.). 
Cotin Didier, boulanger, r. Casimir-Périer, 18.

Blanc Corinne, fleuriste, av. Félix-Faure, 172.

Manificat Pierre, éducateur, Neuville-les-Dames (Ain).

Houver Sabine, docteur médecine, r. Ferdinand-Buisson, 13

Ormancey Gilles, agent technico-commercial, r. Mazenod, 10.

Doudaine Yolande, secrétaire comm., r. Béchevelin, 90.

Guepet Pierre, ingénieur, r. Cino-del-Duca, 31, Paris.

Michel Catherine, étudiante, crs Gambetta, 154.

Falque Claude, chef de fabrication, pl. Docteur-Cinelli, 19, Saint-Symphorien-d'Ozon (Rhône).

Tardy Anne, serveuse-caissière, r. Sainte-Marie, 26.

Donion Serge, agent comm., r. Sainte-Anne-de-Baraban, 8. Pinloche Geneviève, étudiante, r. Ste-Anne-de-Baraban, 8.

Elghozi Rémi, étudiant en droit, all. des Platanes, 12, Antony (Hauts-de-Seine). Cateysson Colette, étudiante en droit, $r$. de Bonnel, 43.

Michel Thierry, maître-nageur-sauveteur, squ. des Violettes, Chassieu (Rhône). Pellegrin Laurence, infirmière, r. Boileau, 288.

\section{QUATRIĖME ARRONDISSEMENT}

Gerber Christophe, agent S.N.C.F., r. Philippe-de-La-Salle, 23.

Monfray isabelle, étudiante, r. Philippe-de-la-Salle, 23.

Mantez Dominique, instituteur, r. Edouard-Millaud, 3.

Mozeret Martine, institutrice, r. Edouard-Millaud, 3.

Fransquin Alex, inspecteur, r. d'Austerlitz, 17.

Janin Laurence, chàrgée d'études, r. d'Austerlitz, 17.

Bernabe Claude, technicien, pl. des Tapis, 4.

Lincio Erika, agent S.N.C.F., "au Bourg» Frans (Ain).

Bouvet Robert, basculeur, r. de la Fraternité, 2, Villeurbanne.

Ciotti Christine, vendeuse, r. du Mail, 16.

Dumas Eric, agent de maîtrise, bd de la Croix-Rousse, 87

Masson Yvette, comédienne, bd de la Croix-Rousse, 87.

\section{CINQUIĖME ARRONDISSEMENT}

Voluzan Bernard, agent recouvrement, r. des Aqueducs, 343.

Rivollier Sylvie, agent hospitalier, Duerne (Rhône).

Martin Xavier, s.p., Entrechaux (Vaucluse). Berthelot Catherine, s.p., r. Pierre-Valdo, 76.

Champ Jean-Claude, empl. de banque, Francheville (Rhône). Veysseyre Joëlle, empl. de banque, r. des Aqueducs, 77.

Malosse Michel, empl. de commerce, bd des Castors, 79. Brunier Véronique, préparat. en pharmacie, Villeurbanne.

Tissler Jean, mécanicien, Le Creusot (Saône-et-Loire). Boca Nathalie, auxiliaire comptable, r. Saint-Georges, 78.

Dumont Jean-Marc, agent commercial, Dommartin (Rhône). Duchemin Marie, étudiante, av. de Ménival, 10.

Après une courte période d'amusement sceptique, la classe entière manifeste un franc intérêt, durable: la monotone, indigeste ou futile liste officielle, jamais vraiment «lue», va révéler à portée de main et d'analyse, des richesses socio-culturelles insoupçonnées et incontournables, va permettre de précipiter des apprentissages socio-scolaires importants et variés. 


\section{LES PROMESSES OFFICIELLES DE MARIAGE}

\section{Construction d'un corpus}

Le texte «en liste» pose ici peu de problèmes techniques de lecture (" ça se répète, c'est toujours pareil, un homme et une femme... ") ; à l'inverse apparaît clairement que lire c'est (anagrammatiquement et fonctionnellement) lier, relire, relier.

Bref, la construction d'un sens sera le produit d'une double opération, maîtrisée: a) constitution d'un texte en objet d'étude et b) interrogation méthodique.

II s'agit donc, d'abord, d'expliciter avec les élèves la notion de corpus afin que les résultats du travail d'analyse soient exploitables et éventuellement transférables.

Cette première opération est éminemment formatrice car, à la différence des (csupports » scolaires de lecture (livres de lecture vs lectures de livres) qui privilégient les discours pré-découpés et les questions pré-établies, elle conduit les apprenants à réfléchir, nécessairement, aux conditions de validité de l'étude.

Les critères fondamentaux d'homogénéité (spatio-temporelle et thématique) d'exhaustivité (les 172 promis) et de représentativité (2) sont progressivement co-dégagés.

Cette appropriation active, collective et explicite d'une démarche «scientifique») appliquée à l'analyse du discours forme l'esprit critique, prépare au travail autonome et permet à chaque élève de se situer par rapport aux véritables exigences du travail intellectuel.

\section{Une méthode d'analyse: l'apport sociologique.}

Le corpus validé, il reste à mettre au point des procédures d'analyse qui permettent de répondre à la question massive que nous nous sommes donc posée: "rqui épouse qui, aujourd'hui, en France?".

Comment «traiter » ce document officiel qui donne trois types d'informations sur les futurs conjoints: nom et prénom, profession et adresse ? En coopération avec la conseillère d'orientation (et en relation avec les problèmes d'orientation) les élèves $s \in$ familiarisent avec la lecture-écriture de la littérature administrative et/ou sociologique (statistiques, tableaux à double entrée, schémas, pourcentages, etc...).

Les brochures de l'ONISEP et les informations apportées par la conseillère permettent de maîtriser peu à peu les notions de classes sociales, catégories socio-professionnelles (C.S.P.) et types d'activités. Le professeur de français lui, apprend d'abord à décoder les abréviations conventionnelles (crs = cours, s.p. = sans profession, etc...) ; il apprendra ensuite à lire, à travers les termes d'identité (voir infra), l'appartenance ethnique et l'intégration culturelle.

(2) Le professeur de géographie peut intervenir pour affiner ce dernier critère en comparant les caractéristiques générales de la population, en France, et les traits dominants de la population lyonnaise. 
Il va sans dire que l'appropriation de l'ensemble de ces notions, commandée par l'effectuation de la tâche, se fait en réalité dans le mouvement même des questionnements successifs et dans l'essai de formulation de constantes sociologiques. En effet la réflexion, soutenue ou non par des lectures commentées de textes théoriques (voir nos notes), relancée par des retours verbalisés ou non sur les représentations spontanées de chacun, permet d'engager une lecture sociologique, certes élémentaire, mais claire, cohérente et appropriée, à tous les sens du terme.

A. Les indications d'appartenance socio-professionnelle permettent d'avancer que de fait, sauf exception rarissime, on se marie dans sa classe (bourgeoisie, classes moyennes, classes populaires), voire dans sa (c caste ) $\left(2^{\mathrm{e}}\right.$ arrond., Roy de Lachaise + Michon du Marais), que souvent les promis travaillent dans le même secteur (primaire, secondaire, tertiaire), parfois dans la même branche professionnelle (infirmier + infirmière, employé de banque + employée de banque, etc...) (3).

La règle dans la formation des unions matrimoniales prend l'allure d'une loi sociologique qui heurte de plein fouet, non pas la réflexion (" Oui, bien sûr, c'est vrai, c'est normal... ") mais les croyances sociales de chacun. Autrement dit, l'idéologie romantique qui assimile amour à liberté ("r L'Amour est aveugle ", " L'amour est enfant de Bohême, il n'a jamais connu de loi" etc...) et mariage d'amour (" On se marie parce qu'on s'aime... ») est brutalement remise en cause. Mais dans la mesure où l'enseignant partage fermement l'idée que "le discours de la science ne peut paraître désenchanteur qu'à ceux qui ont une vision enchantée du monde social ") (4) une double attitude semble souhaitable pour éviter l'obscurantisme comme le terrorisme.

Une première approche, ludique et peu globalisante, tempère les possibles conflits psycho-cognitifs. II s'agit de faire prendre conscience aux élèves qu'ils maîtrisent déjà, en fait, cette "mécanique» matrimoniale : d'abord collectivement, et sur le ( dos ») des profs, dont les élèves s'apercoivent vite, non sans plaisir, qu'ils n'échappent pas à la règle, de ce point de vue du moins : ensuite par petits groupes, en essayant de deviner (pré-dire) à l'annonce du promis, les caractéristiques socio-professionnelles de la promise. Les élèves adorent : ils gagnent souvent! Encore, individuellement, sous forme d'enquête par exemple, en repérant (en vue d'une invite au jeu réel en classe ou hors classe) les jeux de cartes ou d'enfants où l'on cherche à faire des «mariages ») et d'en étudier les modalités (ainsi au "(mariage de rencontre", au "mariage sur table "), au "mariage " tout court l'atout majeur est la réunion, dans la main d'un même joueur, d'un roi et d'une dame de même couleur...).

Une deuxième approche, plus intellectuelle, essaiera de faire travailler sur les théories des sociologues du mariage, de situer historiquement la naissance de l'idée romantique du mariage, bref de relativiser nos pratiques culturelles et de faire apparaître que, de toute manière, les déterminismes sociaux sont peut-être d'autant plus efficaces que méconnus et éventuellement subis individuellement...

(3) Historiquement il y a indiscutablement “régression de l'endogamie professionnelle». Voir par exemple E Shorter, Naissance de la famille moderne, Ed. du Seuil, 1975, pp. 187-192.

(4) P. Bourdieu, Actes de la recherche en sciences sociales, 1975, 1, p. 2. 
On s'appuiera sur l'idée que « la prime éducation, renforcée par toutes les expériences sociales, tend à imposer des schémas de perception, en un mot des goûts qui s'appliquent, entre autres objets, aux partenaires sexuels et qui, en dehors même de tout calcul proprement économique ou social, tendent à écarter la mésalliance: l'amour heureux, c'est-à-dire l'amour socialement approuvé, donc prédisposé à la réussite, n'est autre chose que cette sorte d'amor fati, cet amour de son propre destin social, qui réunit les partenaires socialement prédestinés par les voies apparemment hasardeuses et arbitraires d'une élection libre » (5).

Enfin, toute une série de modalisations sont à apporter, sans détour, aux données et conclusions fournies par le corpus:

a) des facteurs secondaires d'âge (vieux/jeunes), de famille (aîné/cadet), de religion, de "morale") (veuf, divorcé...), de beauté jouent un rôle dans la mesure où ils affectent la valeur d'un individu sur le marché matrimonial sensible au capital économique mais aussi symbolique. Des règles macro-structurales ne sauraient rendre compte de " la diversité infinie des pratiques et, en particulier, des stratégies permettant de concilier, d'équilibrer et parfois d'annuler les contraintes $)(6)$.

b) le corpus donne un état synchronique et limité, un bref instantané, d'une réalité sociale complexe : il est muet sur la trajectoire sociale des familles respectives (7), sur la classe d'origine des futurs époux, sur le volume et la structure du capital (économique et symbolique) des promis, sur la profession des pères, etc... Autant de traits qui permettent à la sociologie du mariage de construire des modèles beaucoup plus fins.

On sait aussi, par exemple, qu'en milieu rural la problématique du mariage présente certaines particularités (exode, célibat, "marché ») restreint, interconnaissance, etc...).

c) le corpus permet de préciser enfin que l'homogamie sociale (8) est particulièrement forte dans les fractions les plus élevées des classes dominantes (9) et chez les plus exclus des classes dominées, peut-être pour des raisons opposées : pour les uns il s'agit de maîtriser les mécanismes de sélection et de reproduction, alors que les autres reflètent des mécanismes d'exclusion (10).

De toute manière, si la confrontation au réel, tel que la sociologie le construit, est insupportable à tel ou tel élève, il est plus qu'inutile d'insister : il faut alors laisser la porte des rêves ouverte à l'exploitation fantasmatique des exceptions, soulignées par leur retentissement médiatique. Grâce entre autres à leur capital esthétique, la Bégum, ex-ballerine italienne, n'a-t-elle pas effectivement épousé, jadis, l'Aga Khan? Nadine Lhospitalier, «père inconnu et mère ouvrière, élevée à Puteaux dans un deux-pièces obscur » ne s'est-elle pas métamorphosée en baronne de Rothschild, épouse de Edmond de Roths-

(5) P. Bourdieu, "Les stratégies matrimoniales dans le systeme de reproduction », in Annales, Economies, Sociétés, Civilisations, no sp., Famille et societé, 4-5, juillet-octobre 1972, p. 1124

(6) Id., ibid.

(7) Y. Delsaut, Le double mariage de Jean Célisse, in Actes de la recherche... 1976, no 4, pp. 4-20.

(8) Un mariage est dit "homogame" (vs "hétérogame») lorsque les conjoints sont issus d'un même milieu (social, economique, culturel...).

(9) M. de Saint-Martin, Les stratégies matrimoniales dans l'aristocratie, notes provisoires, in Actes de la recherche... no 59, sept. 1985, pp. 74-77.

(10) A. Desrosières, Marché matrimonial et structures des classes sociales, in Actes de la Recherche... mars-avril 1978, no 20-21, pp. $97-107$ 
child, "le plus riche de tous les Rothschild"? (11). D'ailleurs, moins spectaculaire mais plus proche, dans le corpus lyonnais lui-même, deux ou trois femmes issues des classes populaires épousent, semble-t-il, des hommes de la classe supérieure...

B. Il apparaît également à la lecture que, comme grand-père, on continue de se marier, presque toujours, lorsqu'on a un métier ( 3 exceptions/86 promesses); de plus, il ne se présente aucun cas d'homme sans profession (s.p.) qui se propose de convoler en justes noces avec une femme qui a un travail. Or l'inverse (femme s.p. + homme ayant un métier) se produit (9 cas).

Ainsi, sur un fond d'homogamie sociale très prononcée se détache, très lisible, une autre caractéristique secondaire mais intéressante des mariages contemporains: dans le couple, le poids économique du mari n'est qu'excep tionnellement inférieur, du moins au moment du mariage, à celui de sa femme. Autrement dit les hommes ne se marient, tendanciellement, que lorsqu'ils ont un métier et n'ont jamais de métier inférieur à celui de leur femme.

Donc, malgré l'accent mis aujourd'hui sur «sa vocation affective»), le mariage n'intervient que «lorsque /'homme et la femme ont acquis l'essentiel de leur formation professionnelle " (12) et la dissymétrie socio-professionnelle à l'intérieur du couple " met en relief une articulation fondamentale entre structures familiales et structures économiques" (13).

C. Le corpus est interrogeable encore en termes d'exogamie/endogamie (14) matrimoniale : conformément à des tendances séculaires les promis vivent dans un espace géographique rapproché (rue, quartier, ville, conurbation, département le plus souvent); on sait par ailleurs que de subtils "rmécanismes sociaux tendent à reconstituer dans les lieux d'arrivée les groupes de départ" (ex. les auvergnats de Paris ou les italiens de Lyon) (15). Dans l'ensemble l'endogamie (ou homogamie géographique) est moins marquée à mesure qu'on s'élève dans la hiérarchie sociale et surtout à mesure qu'on passe des milieux ruraux aux milieux urbains. L'exogamie (ou hétérogamie géographique) est donc rare et ne concerne en effet (mis à part le problème des mariages interethniques) qu'un seul couple d'intellectuels appartenant aux fractions dominantes ( $5^{\mathrm{e}}$ arr., Z.M., géographe, Zurich + J.R., conseil en entreprise, Lyon).

Cette nouvelle constatation soulève de multiples questions chez les élèves: les formes modernes de communication, l'uniformisation étatique des marchés, l'effondrement de la dépendance parentale et lignagère, la mobilité professionnelle, la privatisation de la sphère sentimentale, etc... ne devraientelles pas produire, presque mécaniquement, des unions plus exogames? En fait l'espace où se réalisent les rencontres qui préludent au choix du conjoint est le lieu (et l'objet) de savoirs et de pouvoirs, maîtrisés et balisés très différemment selon les sujets sociaux.

(11) N. de Rothschild, La baronne rentre à cinq heures, Livre de poche no 6097 . Les citations sont extraites de la quatrieme de couverture.

(12) J. Kellerhals et alii, Mariages au quotidien, inégalités sociales, tensions culturel/es et organisation familiale, Collection Regard sociologique, Ed. P.M. Favre, 1982, p. 67

(13) Op. cit., p. 80

(14) Le phenomène, assez important et marqué dans notre corpus, de cohabitation pré-maritale brouille évidemment le repérage des origines géographiques de la famille d'appartenance; on connaît cependant la forte homogamie des couples qui vivent en union libre (voir P.A. Audirac, Cohabitation et mariage, in Economie et statistiques, no 145, juin 1982).

(15) A. Girard, Le choix du conjoint, une enquete psycho-sociologique en france, travaux et documents, INED, cahier no 70, 1974, pp. 189.191 
Ainsi les phénomènes d'interconnaissance sont-ils à rapporter à la structure des divers champs de "rencontre") (les bals, les relations de travail ou d'études, les relations de voisinage enfin prédisposent au choix d'un conjoint spécifique) et à la position de chaque futur(e) sur ce champ : «certes un jeune membre de l'enseignement, célibataire, ne choisit pas le lieu de son affectation, mais une fois nommé, il fréquente d'abord et essentiellement, dans sa nouvelle résidence, les membres de l'enseignement et leurs familles. Ainsi se constituent bien des unions entre membres du corps enseignant, quand elles ne résultent pas déjà de relations plus anciennes de famille, ou d'école") (16).

Autant de pistes de réflexions (ou d'enquêtes: "Comment vous êtesvous connus? ")/ et d'interrogations d'ordre socio-historique et culturel, fort complexes en vérité, qui mobilisent expériences personnelles et culture générale.

D. L'étude précise des patronymes permet enfin d'évaluer le très faible taux de métissage ethnique ou racial. Une passionnante (parfois délicate) réflexion anthroponymique (qui peut être réutilisée sans difficulté dans l'approche de l'onomastique littéraire) amène à conclure rapidement que noms et surtout prénoms, biens symboliques (17), sont des indicateurs puissants d'intégration culturelle. Trois cas se présentent: les mariages franco-français (mathématiquement majoritaires) et les mariages mixtes, sans omettre le cas, fréquent, d'étrangers qui se marient entre eux (Maria Rel Antonio + Da Silva Goncalves Maria, Ayari Jauél + Bouhadjar Dalila).

Les mariages mixtes permettent d'affiner l'analyse: union entre français(e) et francisé(e) (Vincent Dominique + Hernandez Marie), union entre francisé(e)s, couples franco-étrangers (Réa Tonino + Bonod Véronique ; Saighi Djony Faouzi + Fevre Nadine).

A l'opposé des représentations immédiates nourries de romantisme le mariage apparaît donc comme un acte social, socialement réglé sur une homogamie généralisée. N'importe qui n'épouse pas n'importe qui, sans aucun doute parce que ule marché matrimonial est, avec le marché du travail, un des lieux privilégiés où se négocient, se transmettent et se "réalisent" (au sens où on parle de réalisation de la valeur) les divers types "d'héritage " dont disposent les agents " (18). Au total la classe s'est effectivement construit un savoir de référence, simple mais partagé, transférable à d'autres types de textes et articulé sur une réalité vécue sinon connue jusqu'alors. Ce savoir suscite deux questions que nous n'abordons pas directement dans notre classe:

1 - la urelative immobilité sociale des sociétés industrielles contemporaines" (19) est-elle, comme il semble, à mettre en rapport avec le mariage, "fondement de la famille qui, elle-même, est le principal creuset où s'élabore la socialisation des enfants et où prennent naissance et s'enracinent les différences et inégalités sociales ultérieures")? (20).

(16) Id., ibid., pp. 192-197

(17) Sur la valeur identitaire des systemes d'appellation on peut lire L'Homme, octobre-décembre 1980, tome XX. no 4 et Le Prénom, mode et histuire. Les entretiens de Malher, 1980. École des hautes études en sciences sociales, 1984.

(18) A. Desrosieres, op. cit., p. 97

(19) A. Girard, op. cit., p. 28.

(20) $\mathrm{Cl}$. Thélot, Tel père, tel fils? position sociale et origine familiale, Dunod, 1982, pp. 187-188. 
2 - « hier comme aujourd'hui, toutes les recherches historiques, démographiques, ethnologiques confirment la règle homogamique " (21); mais " hier cette règle était explicite voire acceptée alors qu'aujourd'hui il y a conflit implicite entre des pratiques homogamiques et des discours hétérogamiques. Pourquoi l'idéologie installe-t-elle entre le mariage dans les sociétés traditionnelles et les formes matrimoniales contemporaines une contradiction et, conséquemment, pourquoi le sens commun méconnait-il l'omni-prégnance des codes sociaux qui socialisent le sentiment amoureux et reconnait-il la force romantique et transcendante de l'amour?") (22). Peut-être pour ne pas découvrir que l'amour est conservateur...

\section{PROMESSES (TENUES) DE LECTURES-ÉCRITURES.}

Le travail sur le «Bulletin... »), document à la fois officiel, simple et « utilitaire $\gg$ a donc facilité des apprentissages de divers ordres. La méthode de lecture (en tant qu'initiation à la pratique du corpus, questionnement précis et motivé du texte, prélèvement concerté et organisation systématique d'indices) a été affermie. Le «Bulletin... »), propriété commune, a permis de mettre en place un rapport non sacralisé à l'objet d'étude, de mettre les élèves en confiance et de faire saisir que le sens est partout... à saisir. Le discours administratif se prête enfin plus difficilement que la littérature à des jugements de valeur aussi immédiats qu'arbitraires; il favorise au contraire une approche strictement descriptive (ici sociologique) qui met à l'aise les élèves.

Nous allons voir que, dans la logique interne de notre travail, cette disposition est précieuse car elle tend à minorer les lectures éternellement psychologisantes et/ou non critériées, à mettre en cause la coupure nette entre écrits fictionnels et non fictionnels, entre textes légitimes et non légitimes, à donner des repères d'écriture plus rigoureux.

\section{A. LE PROSCRIT}

Notre lecture des promesses de mariage permet de dégager le négatif du texte, de le lire en creux, de sensibiliser par un exemple précis et concret à la problématique du non-dit, qui bien souvent permet en fait de ((penser ») le dit. On sait par ailleurs combien (et pas toujours à tort) les élèves gardent en général leurs distances (fût-ce intérieurement) quand le professeur développe les implicites du texte, notamment ses présupposés culturels.

\section{1) La prohibition de l'inceste}

Turbelin Olivier n'épouse, en aucun cas, Turbelin Michèle (sœur, mère ou fille). C'est dire que d'autre lois - à tous les sens du terme - entrent en jeu.

Un amusant retour au folklore enfantin autorise la discussion de ce sujet tabou et fait découvrir les subtilités de l'écriture poétique des comptines:

(21) M. Segalen, Sociologie de la famille, A. Colin, 1981, p. 110.

(22) J. Kellerhals et alii, op. cit., p. 69. 
«Bonjour ma cousine

Bonjour mon cousin germain

On m'a dit que vous m'aimiez, est-ce bien la vérité?

Je m'en soucie guère, je m'en soucie guère, passez par ici

Et moi par là

Au revoir ma cousine et puis voilà!'"

Ce petit texte chantonné (en chœur!) mime, sur un rythme gai, l'évitement des rapport incestueux. Le style dialogué, l'ambivalence des formes possessives, la généralité des rapports de parenté, le désir interrogé, la prise en charge de l'interdit, l'identification aisée au jeu, la formulation de la Loi en termes métaphoriques, la solution dédramatisée du conflit latent donnent à lire le procès d'une socialisation réussie des affects, chez le petit garçon comme chez la fillette.

Il va de soi qu'on aurait pu aussi bien rappeler telle tragédie antique ou racinienne, conter Peau d'Ane, analyser en détail les rapports troublants d'Eugénie Grandet et de son cousin Charles Grandet, auditionner tel zeste de chanson de Gainsbourg, etc... et faire écrire un petit texte où l'on s'efforce de travailler l'art du sous-entendu.

Selon les niveaux on peut aborder, dans une perspective toujours claire et maîtrisée, d'autres textes encore (mythes ou récits populaires), évoquer Fdipe, souligner avec Levi-Strauss que, quelle que soit la complexité des systèmes de parenté, la prohibition de l'inceste est une coercition culturellement productrice puisqu'elle assure la régulation de l'échange. Cette obligation de l'échange est bien sûr variable: quel élève ne voudra faire un exposé sur les " Azandé, dont les nobles se marient avec leurs filles, les Hawaïens, dont l'aristocratie pratique le mariage entre frères et sœurs, les mariages incas ou pharaoniques (...) ) (23).

Cette obligation, universelle, accomplit rien moins que le "r passage de la nature à la culture ») (Levi-Strauss) :

"Tu voudrais épouser ta scur? commentent les Arapesh à l'ethnologue qui les interroge. Mais qu'est-ce qui te prend? Tu ne veux pas avoir de beaufrère? Tu ne comprends donc pas que si tu épouses la scur d'un autre homme et qu'un autre homme épouse ta sœur, tu auras au moins deux beaux-frères, et que si tu épouses ta propre sœur tu n'en auras pas du tout? Et avec qui iras-tu chasser? Avec qui feras-tu les plantations? Qui auras-tu à visiter?") (24).

Deux pistes de réflexion, l'une vite reconnue, l'autre plus tortueuse, peuvent être proposées. Elles visent à faire apparaître des formations de compromis avec la Loi :

a) toute épousée qui, contrairement à la loi mais conformément à nos coutumes, prend le nom de son mari, ne devient-elle pas, ipso facto, et symboliquement (dans l'ordre du langage) le double (patronymique) de la mère de l'époux? De plus, sauf à " habiter en gendre") comme on disait autrefois, la mariee venait vivre sous le toit de sa belle-mère...

Tout le théâtre de Feydeau peut être relu de ce point de vue et une saynète qui joue sur ce type de quiproquo, écrite! 
b) si l'on admet que dans l'économie gènérale des échanges, l'économie des échanges affectifs familiaux travaille aussi, à son niveau, le marché matrimonial, on peut imaginer que le prénom est "l'un des supports privilégiés du déplacement des sentiments incestueux" (25). Un sociologue, s'appuyant sur la thèse freudienne bien connue - « un certain degré de fétichisme se retrouve régulièrement dans l'amour normal") - a pu en effet vérifier "rstatistiquement l'importance des éléments incestueux dans le choix amoureux ") (26) par prénom interposé. Napoléon ne s'est-il pas résolu à épouser Joséphine parce qu'il pouvait ainsi «transférer sur elle une partie du tendre attachement qu'il ressentait pour Joseph, son frère aîné ") (27)?

Une surprenante lecture de l'onomastique romanesque peut s'engager... et un petit récit à clés (celles de l'inconscient !) est mis en chantier, puis proposé à la lecture sagace de chacun.

\section{2) La polygamie interdite}

Turbelin Olivier n'épouse pas, en même temps, Lerouge Michèle et Dor Christine. Cette fois c'est la loi française qui le lui interdit, expressément.

Une lecture du Code civil peut être alors proposée (28), comme une recherche sur les différents systèmes monogamiques et polygamiques. En $3^{\circ}$ on s'est contenté d'aborder ce point en lisant (ré-écrivant, jouant...) un extrait de Monsieur de Pourceaugnac, de Molière.

Dans cette comédie, écrite en 1669, Monsieur de Pourceaugnac est un gentilhomme provincial à qui une jeune et jolie parisienne est promise en mariage par son vieux père. Mais celle-ci s'oppose par tous les moyens à cette union et son ami Sbrigani imagine un stratagème pour écarter le prétendant: on apprend que Pourceaugnac aurait épousé Lucette à Pézenas et Nérine à StQuentin. Pure fiction, mais notre limousin doit prouver son droit...

\section{Scène $x$ : Monsiew de Pourceaugnac, Sbrigani.}

MONSIEUR DE POURCEAUG.NAC:

Ah! je suis assommé! Quclle peine! Quelle maudite ville! Assassine de tous cotés!

\section{SBRICIANI}

Qu'est-ce, monsieur? Est-il encore arrive quelque chosc?

MONSIEUR DE POURC:EAUGNAC:

Oui. Il pleut en ce pays des femmes et des lavements.

\section{SHRIC}

comment donc:

\section{MONSIEUR IDF POURCFALINAC}

Deux carognes de baragouineuses me sont venues accuser de les avoir épousées toutes deux. et me menacent de la justice.

\section{SBRIC $A A N I$}

Voila une méchante affairc : et la justice, en ce pays-ci, est rigoureuse en diable contre cette sorte de crime.

MONSIEUR DE: POURCEAUG INAC:

(rui: mais quand il y aurait information, ajournement, décret, et jugement obtenu par surprise, défaut et contumace, jai la voie de conflit de juridiction pour temporiser. et venir aux moyens de nullité qui seront dans les procedures.

\footnotetext{
(25) B. Vernier, Stratégies matrimoniales et choix d'objet inc'stueux, in Actes de /a recherche, 57-58, 0.27 (26) Id., ibid., p. 25.

(27) Id., ibid., (citation de Freud).

(28) J. Costa-Lascoux, Lire le drait, un enjeu social?, Le Français aujourd'hui, no 57, mars 1982, pp. 57-63.
} 


\section{SBRIGANI}

Voila en parler dans tous les termes ; et l'on voit bien, monsieur, que vous êtes du méticer.

\section{MONSIEUR DE POURCEAUGNAC:}

Moi! point du tout. Je suis gentilhomme.

\section{SBRIGANI}

Il faut bien, pour parler ainsi, que vous ayez. etudie la pratique.

\section{MONSIEUR DE POURCEAUGNAC;}

Point. Ce n'est que le sens commun qui me fait juger que je serai toujours reçu a mes faits justificatifs, et qu'on ne me saurait condamner sur une simple accusation, sans un récolement et confrontation avec mes parties.

\section{SBRIGANI}

En voila du plus fin encore.

\section{MONSIEUR DE POURCEAUGNAC}

Ces mots-la me viennent sans que je les sache.

\section{SBRIG;ANI}

I me semble que le sens commun d'un gentilhomme peut bien aller a concevoir ce qui est du droit et de l'ordre de la justice, mais non pas a savoir les vrais termes de la chicane.

\section{MONSIEUR DE POURCEAUGNAC;}

Ce sont quelques mots que j'ai retenus en lisant les romans.

$$
\text { SBRIGANI }
$$

Ah! fort bien!

\section{MONSIEUR DE POURCEALGNAC:}

Pour vous montrer que je n'entends rien du tout a la chicane, je vous prie de me mener chez quelque avocat, pour consulter mon affaire.

\section{SBRIC;ANI}

Je le veux, et vais vous conduire chez deux hommes fort habiles ; mais j'ai auparavant a vous avertir de n'etre point surpris de leur manière de parler ; ils ont contracte du barreau certaine habitude de déclamation qui fait que l'on dirait qu'ils chantent, et vous prendrez pour musique tout ce qu'ils vous diront.
MONSIEUR DE: POURCEALGNAC.

Qu'importe comme ils parlent, pourvu qu'ils me disent ce que je veux savoir!

Scène XI: Monsieur de Pourceaugnac, Sbrigani, deux Avocats, deux Procureurs, deux Sergents.

PREMIER AVOCAT, trainant ses paroles en chantant.

Ia polygamic est un cas, Est un cas pendable.

SECOND AVOCAT, chantant fort vite et en bredouillant,

Votre fait

Est clair et net ;

Et tout le droit,

Sur cet endroit,

Conclut tout droit.

Si vous consultez nos auteurs,

Législateurs et glossateurs,

Justinian, Papinian,

Ulpian, et Tribonian,

Fernand, Rebuffe, Jean Imole,

Paul, Castre, Julian, Barthole, Jason, Alciat et Cujas,

Ce grand homme si capable:

la polygamic est un cas Est un cas pendable.

\section{ENTREE DE BALLEYI}

Danse de deux procureurs et de deux sergents, pendant que le second avocat chante les paroles qui suivent:

'Tous les peuples policés Et bien sensés,

Les Français, Anglais, Hollandais,

Danois, Suedois, Polonais,

Portugais, Espagnols, Flamands, Italiens, Allemands,

Sur ce fait tiennent loi semblable:

Et l'affaire est sans embarras,

Ia polygamic est un cas, Est un cas pendable.

I.E PREMIER AVOCAT chante celle-ci:

Ia polygamic est un cas, Est un cas pendable.

Monsieur de Pourceaugnac, impatienté, les chasse. 
M. de Pourceaugnac va être la victime nécessaire d'un étonnant charivari. Les règles de l'alliance homogamique ne sont-elles pas violées? La mésalliance envisagée par le barbon n'est-elle pas aussi absurde, scandaleuse et d'un certain point de vue, «fantaisiste», qu'un mariage polygamique? La dérision charivarique ne s'attaque-t-elle pas par prédilection au remariage des veufs (ou veuves) en tant que la «(polygamie latente)) de ce type de (ré-)union contredit la «monogamie explicite», norme triomphante! (29).

Il est alors aisé de construire avec les élèves le «carré » sémiotique suivant, matrice de lectures-écritures fructueuses:

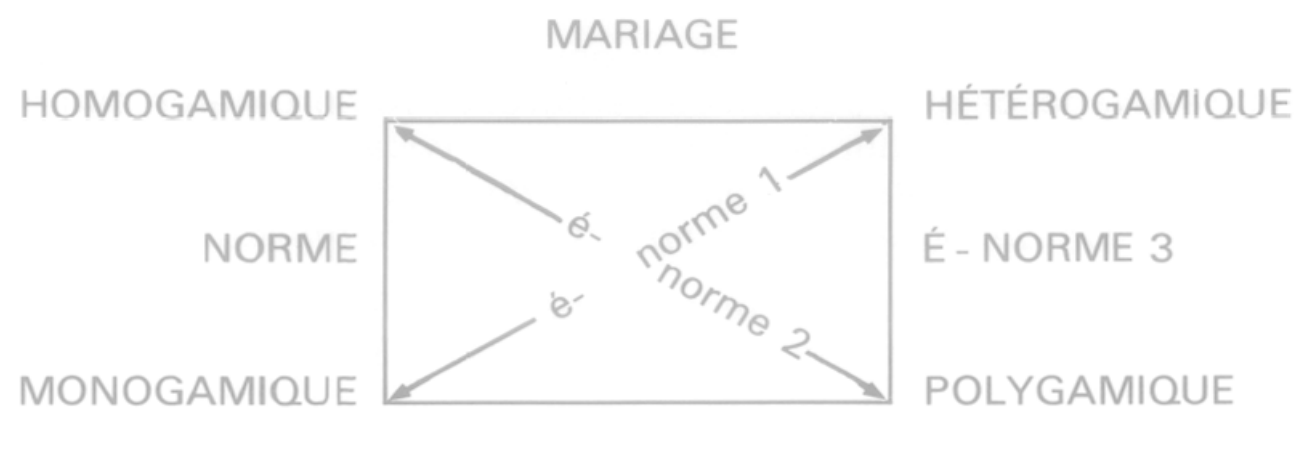

Il est clair que d'innombrables héros (ou héroïnes) de fiction peuvent être «lus » ou «construits») selon l'un ou l'autre de ces axes, surtout si l'on y ajoute l'adultère, ıdémenti le plus éclatant, le plus grossier de la norme monogamique » (30).

Si la polygamie, inventée, vaut et/ou s'ajoute à l'hétérogamie, projetée, $M$. de Pourceaugnac est menacé de commettre deux (rénormités». Et si la polygamie est bien, selon une logique farcesque, la mise à nu de l'hétérogamie, les élèves sont désormais en mesure de saisir l'un des enjeux fondamentaux de la pièce et de repérer aisément les phénomènes:

- d'exogamie: dès l'arrivée de Pourceaugnac le mot (étranger » est prononcé par Sbrigani (!) et dès I, 1 le thème de l'endogamie est marqué par Nérine: "S'il a envie de se marier, que ne prend-il une Limosine (...) ?") 47 ans).

- d'écart d'âge : le prétendant est visiblement âgé (Molière créa le rôle à

- d'écart de fortune : ce provincial est perçu comme un arriviste. Sa fortune comme sa prétention à l'aristocratie sont suspectes. II ne saurait épouser une jeune fille de qualité!

- de mixité culturelle: que «ne laisse-t-il en repos les Chrétiens ?» $\mathrm{M}$. de Pourceaugnac serait sur le point d'épouser une troisième femme et aucune de ces trois femmes ne correspond à son statut social : tout concourt à ce que le groupe des jeunes qui doit assurer le contrôle de l'échange des femmes rappelle le droit sexuel. Les violences rituelles de type charivarique s'abattant en effet sur notre homme, jusqu'à son exclusion coutumière.

(29) Cl. Karnoouh, Le charivariou I'hypothese de la monogamie, in Le charivari, actes publies par J. Le Goff et J.- C. Schmitt, Paris, 1981, pp. $33-43$.

(30) ld., ibid. "r'adultere ne bouleverse pas uniquement la norme monogamique, il ebranle tous les statuts et les róles établis dans le cours des noces : /'homme marié s'y approprie le role des celibataires, la femme mariée ris. que les conséquences d'une procréation illegitime, le jeune celibataire se substitue à l'homme marie et la jeune fille se transforme en pseudo-veuves, p. 42. 
Une mise en perspective historique fait rapidement découvrir les références aux menaces militaires des polygames turcs si redoutées à cette époque, les allusions voilées aux favorites royales. Une approche psycho-culturelle permet de souligner les jeux avec les codes culturels et ses interdits, le theme du monde à l'envers. Une recherche plus littéraire montre comment la polygamie, alliance insensée (31) est transposée dans l'écriture même de la pièce: nom du personnage principal $(M$. de + pourceau), positions incongrues des personnages, bizarrerie des expressions (《pleuvoir des femmes»), discours incontrôlé, registre inhabituel de la parole (Loi chantée), bruits dans la communication (bredouillement précipité).

En définitive ce Pourceau-gnac, ce pourceau-puceau, ce naïf et provincial homme sauvage, ce gros cochon de Carnaval va voir proliférer, comme une métaphore développée, autour de lui et en lui, le "rvocabulaire et l'imagerie des obsessions les plus redoutables: obsession de la castration, obsession du cocuage, obsession de la bigamie et obsession de /'homosexualité") (32).

On verra comment cela retentit dans l'écriture des élèves qui pour l'heure jouent avec "les anomalies sémantiques» (33).

\section{3) L'homosexualité inter-dite}

Turbelin Olivier n'épouse pas non plus, enfin, Levera Gérard. Au-delà des condamnations bibliques (Lévitique XX, 3, Rom. I, 26), de David et Jonathan ou des désirables beautés des éphèbes grecs, au-delà des bûchers médiévaux et des dispositions, nuancées, du Code Napoléon, au-delà des problèmes socioculturels de la «condition homosexuelle» et de la problématique psychanalytique, le cadre de la classe autorise l'approche de la dévalorisation de l'homosexualité à travers les injures sexistes que nos élèves connaissent bien. Passé le frisson provoqué par l'impertinence de tels propos, on conviendra que l'inconvenance de l'objet étudiè n'est pas l'inconvenance du chercheur, que ules histoires du diable, le vocabulaire des jurons populaires, les chansons et les coutumes de nurseries, tout cela acquiert une signification ") aux yeux de Freud... comme aux nôtres! (34).

Sans se risquer forcément à un cours de jurologie on s'aperçoit vite que l'information sous-jacente à une phrase du type " $T$ 'es pas un mec, t'es une vraie gonzesse " est énorme (35). Toute injure n'est-elle pas un condensé langagier et un révélateur familier des valeurs fortes et implicites qui structurent une culture ? II est clair ainsi que les termes qui vilipendent l'homosexualité reflètent "l'opprobre jeté sur toute sexualité marginale, non reproductrice et donc anti-sociale (...); la pire injure qu'on puisse de nos jours adresser à un homme est encore une injure misogyne (...) ") (36). L'homosexuel passif, I'homme-objet est d'autant plus haïssable qu'il est censé être r(le sujet par excel-

(31) Levi-Strauss (Le Cru et le Cuit, Plon, Paris, 1964, p. 295) estime que «toute anomalie dans le déroulement d'une chaîne syntagmatique" (matrimoniale) produit un "bruit) que le bruit charivarique a pour rôle de signaler, voire de contre-balancer metaphoriquement.

(32) P. Gayrard. Monsieur de Pourceaugnac: un charivari à la Cour de Louis XIV?, in Le Charivari, pp. $309-317$

(33) L'arlicle de T. Tudurov, Les anumalies sémantiques, in Langages, no 1, 1966. pp. 100-123, uffre de numbreuses possibilites d'application en ce sens.

(34) S. Freud, La naissance de la psychanalyse, P.U.F., 1973, p. 167.

(35) L.-J. Calvet, Pour et contre Saussure, Payot, 1975. pp. 137-145

(36) N. Huston, Dire et interdire, Payot, 1980, p. 57 
lence " (37). C'est dire que l'insulte sexuelle est "strictement à sens unique") (38) et que la ('langue du mépris») renvoie bien une certaine image de la société et des rapports de force qui la régissent. On étudiera par exemple «les noms du pédéraste passif qui forment une série extrêmement riche ") tant l'idéologie phallocrate est puissante (39).

D'une manière générale on peut assurer que les élèves se plaisent à compter ces "coups à valeur sociologique" que sont les insultes et à en reconstruire la (〈logique») culturelle dans une œuvre littéraire donnée: injures carnavalesques chez Rabelais, injures homosexuelles dans la thêâtre médiéval des soties, litanies injurieuses d'un Marot, injures hugoliennes de la Fête des Fous (Notre-Dame de Paris), injures populaires dans les romans de Zevaco ou de San Antonio, injures surréalistes (40) injures rituelles dans La Guerre de Troie... ou dans La Guerre des boutons, etc...

Peut-être s'en tiendra-t-on à l'écriture d'un petit texte d'injures-fiction en appliquant la "Grammaire des insultes" (41) et en s'inspirant des "Gros mots des enfants" (42) ou de la rhétorique épique: "ren tout domaine la chanson de geste recourt à l'hyberbole. Elle aime les injures énormes et les coups d'épée formidables (...). Presque tous les filz a putain de l'épopée ne sont pas "justifiés") par la situation" (43)... Culvert de Brest!

\section{B. LE PRESCRIT}

\section{1) Le mariage, malgré tout}

Certes des restrictions pèsent sur la conclusion des mariages et les français(es) se marient moins, alors que l'union libre est de plus en plus courante. Cependant, malgré cette perte de vitesse le mariage reste une pratique (sinon une institution) encore solide : en France on célèbre un mariage toutes les deux minutes (334.000 en 1982, 300.000 en 1983, 284.000 en 1984); de plus il a été établi que la cohabitation, juvénile ou non, obéit aux mêmes règles sociales que la constitution des couples "légitimes» (voir supra).

a) Perte de prestige.

Une fine étude des messages médiatiques peut être conduite avec pour fil directeur, simple, la thèse «officielle» suivante: «Naguère, toute une série de productions (films, romans, nouvelles, opérettes, pièces de théâtre) s'achevaient par une " happy end», une fin heureuse symbolisée, comme dans les contes de fées, par un mariage. II n'y a guère que dans les romans-photos qu'aujourd'hui on pourrait trouver ces dénouements. Dans tous les autres contenus de fiction, et la télévision a ajouté sa puissance de suggestion des "modèles", le mariage apparaît plutôt comme source de conflits que comme but privé et social. Toutes les analyses de contenu des médias confirment ce dénigrement devenu classique " (44).

(37) P. Guiraud, Sémiologie de la sexualité, Payot, 1987, pp. 100-105.

(38) M. Yaguello, Les mots et les femmes, Payot, 1980, pp. 149-163.

(39) P. Guiraud, Dictionnaire érotique, Payot, 1978, p. 78.

(40) R. Édouard, Dictionnaire des injures, Tchou, 1967.

(41) N. Ruwet, La grammaire des insultes, Éd. du Seuil, 1982, pp. 239-316

142) P. Boumard, Les gros mots des enfants, Stock, 1979.

(43) Ph. Ménard, Le rire et le sourire dans le roman courtois au Moyen-Age (1150-1250), Droz, Genève, 1969, p. 237

144) Le statut matrimonial et ses conséquences juridiques, fiscales et sociales, rapport présenté au nom du Conseil économique et social par E. Sullerot, 31-01-1984, p. 31. 


\section{b) Persistance d'une pratique.}

Le (dé)montage ludique d'une page de catalogue pro-nuptial ou la lecture-écriture de petites annonces matrimoniales permet de faire jouer les savoirs acquis, et de les affiner. Les petites annonces du Chasseur francais par exemple, strictement monogames et hétérosexuelles, obéissent à une rhétorique qui traduit des enjeux conventionnels et des représentations culturelles dominantes. II s'agit, entre autres, de concilier la présentation intéressée de soi et la demande d'affection matrimoniale, de sélectionner dans l'offre comme dans la demande les traits "convenables» les plus conformes à l'image que l'on se fait des attentes du lecteur (de la lectrice) à convaincre, de dire sans dire en jouant de la valeur connotée de certains mots («biens», «situation »...); aussi constate-t-on que là encore l'homogamie domine très largement, et sous des formes convenues: les femmes mettent l'accent beaucoup plus et beaucoup plus souvent sur leurs qualités esthétiques et relationnelles, les hommes sur leur "(poids » économique. On retrouve même la forte tendance endogamique des mariages "classiques» puisque la localisation géographique du demandeur vient très souvent en tête de sa proposition. Une étude comparée (Chasseur français / Libération) met en relief le poids des différents types de lectorat sur le «message»; une étude diachronique peut évaluer l'évolution des stéréotypes de (re)présentation et essayer de confronter les critères de séduction chez Delly et dans tel roman de la littérature sentimentale contemporaine. Dans le même ordre d'idée on fera écrire l'annonce matrimoniale de Panurge ou celle d'Emma Rouault...

\section{2) L'homogamie, toujours...}

I.OU MOUSSL E LA PAISANTE

- *Bonjour, belle paysanne.

Paris n'enfante pas,

(bis)

Parmi nos belles alames.

De si puissants appas.

(bis)

- Moussu. quino hardicsso. Quin frount, batz disc qu'éi Mès, a bostos caressos.

Jamès sucoumberèi.

- Moussu, n'è que misèro.

Talo sous mes brassotz.

Mous pés dens la poussiéro,

Ou deguem bieills esclops.

- Dans vos jupes de toile,

Vous semblez dans l'azur

Une brillante étoile,

Sur un nuage obscur.

Voilà des pierreries,

Puis, acceptez cet or.

Ces chaines si jolies.

Eblouissantes d'or.

- Dèchatz-me a la segado,

Dab' lous segadous.

(bis)

Jou me soui reserbado.

Per d'autes segadous. -

(bis)

\section{L.E MONSIEUR ET IAA PAYSANNF}

- Bonjour, belle paysanne.

Paris n'enfante pas.

(bis)

Parmi nos belles dames.

De si puissants appas.

(bis)

- Monsieur, quelle hardiesse.

Quel front, direz-vous que $j$ 'ai.

Mais à vos caresses.

Jamais je ne succomberai.

- Monsicur, je n'ai que misère

Voyez suer mes petits bras.

Mes pieds dans la poussière,

Ou dans de vieux sabots.

- Dans vos jupes de toile.

Vous semblez, dans l'azur

Une brillante etoile.

Sur un nuage obscur.

Voilà des pierreries.

Puis, acceptez cet or.

Ces chaines si jolies.

Eblouissantes d'or.

- Laissez-moi a la moisson.

Avec les moissonneurs.

(bis).

Je me suis réservée,

Pour d'autres moissonneurs (1).

(bis).

(1) Dicté par Françoise Lalanne, de Lectoure. (f. Lamarque de Plaisance, 67-68 (Bazadais). 
Cette chanson de mise en garde (45), dont le thème est extrêmement fréquent dans le folklore d'autrefois, repose explicitement sur le principe du refus, poli, de I'hétérogamie: comme dit le proverbe populaire "Amour de Monsieur, eau dans le panier"s.

Paris s'oppose à province, comme Monsieur à paysanne et français à garçon. La "(belle paysanne " sait qu'aux yeux du Monsieur son capital esthétique ne suffit pas et que tout ce qui brille n'est pas or... Il est intéressant de comparer ce discours d'auto-conservation sociale à celui de Figaro ou aux doléances, plus «féministes » que des femmes du Tiers-État adressent au Roi ( $1{ }^{\text {er }}$ janvier 1789):

"Les femmes du Tiers-État naissent presque toutes sans fortune ; leur éducation est très négligée ou très vicieuse (...). Les premiers devoirs de la Religion remplis, on leur apprend à travailler; parvenues à l'âge de quinze ou seize ans, elles peuvent gagner cinq ou six sous par jour. Si la nature leur a refusé la beauté, elles épousent, sans dot, de malheureux artisans, végètent péniblement dans le fond des provinces, et donnent la vie à des enfants qu'elles sont hors d'état d'élever. Si, au contraire, elles naissent jolies, sans culture, sans principes, sans idée de morale, elles deviennent la proie du premier séducteur, font une première faute, viennent à Paris ensevelir leur honte (...)" (46).

\section{3) Le patriarcat, bien sûr}

On peut lire de ce point de vue, littéralement et avec pertinence, la mise en page de l'information municipale qui, dans sa disposition même, traduit la " domination » masculine : le nom des futurs ne vient-il pas en premier, celui de la future en second, décalé et sou-mis... ? On remarque alors que dans la même logique patriarcale les enfants légitimes sont nés d'hommes, uniquement!

Selon l'opportunité sont analysés dans cette perspective:

- le récit biblique de la création de la femme (Genèse, 2, 18-25) : ( de la côte qu'il avait tirée de l'homme, Yahvé Dieu façonna une femme "); on sait que selon une tradition hébraïque, le premier homme et la première femme avaient été créés simultanément (Adam + Lilith). II y avait entre eux égalité complète (androgynie?) mais bientôt Adam voulut s'imposer comme chef de famille; Lilith invoqua alors le nom de "l'Ineffable», reçut miraculeusement des ailes et put quitter les jardins d'Eden. Elle séduisit Samaël, le maître des anges déchus et, jalouse, se serait métamorphosée en serpent tentateur... (47).

- une chanson de révolte féministe ; Une sorcière comme les autres"), d'A. Sylvestre:
(...) " i'étais celle qui attend
mais je peux marcher devant
j'étais la bûche et le feu
l'incendie aussi je peux
j'étais la déesse-mère
mais je n'étais que poussière
j'étais le sol sous vos pas
et je ne le savais pas (...)" 1 .

\footnotetext{
(45) J.-F. Bladé, Poésies populaires de la Gascogne, Paris, 1882, T. II. pp. 178-181.

(46) Cahiers de doleances des temmes en 1789, Ed. des femmes, 1981, o. 26

(47) J. Bril, Lilith ou la mere obscure, Payot, 1984
} 
- "Les sabots", conte de Maupassant, dans lequel la fille d'un vieux paysan est livrée corps et biens, comme servante, à Maître Omont. Une réécriture matriarcale, fort récréative et fort éclairante, fait jouer les points de vue en permutant les rapports entre personnages par une simple transposition des marques pronominales (il/elle) ou personnelles (César/Adélaïde):

" Et il gagna sa chambre, une mansarde sous le toit. ll fit sa prière, se dévêtit et se glissa dans ses draps. Mais soudain il bondit, effaré. Un cri furieux faisait trembler la maison: "César?"). Il ouvrit sa porte et répondit, de son grenier: "Me v'là, me v'là - Ousque t'es? - Mais j'suis dans mon lit"s. Alors elle vociféra: "Veux-tu bien descendre, nom de D... et si tu n'veux point, tu vas me foutre le camp, nom de D..." I. Alors, il répondit d'en haut, éperdu, cherchant sa chandelle: "Me v'là, me v'là !).

- L'École des femmes (1662) de Molière, où Agnès apprend d'Arnolphe, "son mari, son chef, son seigneur, et son maître" que le sexe féminin " $n$ 'est là que pour la dépendance" car "du côté de la barbe est la toute-puissance". Agnès n'aurait plus qu'à lire "rles maximes du mariage, ou les devoirs de la femme mariée, avec son exercice journalier") (III, 2)...

- un nouveau corpus, cette fois de proverbes populaires ( $X \mid X^{e} s$.) qui touchent au rôle du père dans le mariage de ses enfants ("relui qui a marié toutes ses filles peut mettre ses mains derrière le dos )/) et aux relations entre époux ("r A la table et au lit la droite appartient au mari»)). De nombreux proverbes parlent en effet, sans détour, de la "sstratégie matrimoniale paternelle. Le mariage règle la place des filles, les faisant passer des mains de leurs pères à celles de leurs époux. Tous les proverbes valorisent la condition masculine: sur le marché matrimonial les problèmes de dot dévalorisent les filles " (48). Ces proverbes "composent un discours masculin") (49) et renvoient à des pratiques matrimoniales et conjugales «apparemment plus soumises aux traditions et aux rituels $\gg(50)$.

Une réécriture de proverbes parodiés ou actualisés permet à chacun(e) de donner la mesure d'un engagement maîtrisé dans une forme très codée. A ce stade du travail le débat d'idées, nourri de lectures et écritures diverses, demeure vif, en effet : la classe prend de plus en plus conscience qu'il n'y a pas de discours (et de pratiques!) innocents, que d'un côté ne trône pas une Culture lointaine, seule digne de réflexion, et de l'autre, «le reste ». II devient clair au contraire que c'est un ensemble de textes différents (par leur statut, leur fonction, leur valeur) qui « informent » (et nous informent sur) un champ culturel ; c'est donc leur mise en relation, et non leur sélection arbitraire, qui est un gage de meilleure appropriation.

Dans ce contexte la manipulation du discours proverbial donne aux élèves le pouvoir de cristalliser des enjeux et des positions (bientôt épinglés sur les murs du collège) et ce d'autant plus aisément que la «structure claire et close » du genre proverbial est facilement didactisable. Soit le proverbe «charivarique $)$,

"Qui vole un veuf, viole un bœuf",

ou le proverbe homogamique

"Qui se ressemble, s'assemble".

(48) M. Segalen, "Le mariage, lamour et les femmes dans les proverbes populaires français», in Ethnologie francaise, 1975, T. 5, pp. 119-162 et 1976, T. 6, no 1, pp. 33-88

(49) Id., ibid.

(50) N. Belmont. Rituels de courtoisie, in Ethnologie française, 1978, VIII, 4, p. 284. 
il est commode de repérer le système des échos phoniques, la binarité rythmique, l'indétermination grammaticale (on, partout, toujours), la valeur métaphorique et connotée, le style archaïsant, la modalité assertive et la logique binaire (relations d'équivalence ou d'opposition) que les différents couplages (phonétiques, morpho-syntaxiques et lexicaux) soulignent le plus souvent (51).

Quatre opérations rhétoriques de base (adjonction, permutation, suppression, substitution, d'une lettre, d'une syllabe, d'un mot, d'un syntagme...) multiplient alors à l'infini les possibilités de réécritures proverbiales (52):

- "Fille libérée, de mari n'a pas nécessité"

"Beau garcon, fleur de mai en un jour perd sa beauté")

"Débarrassée de son tablier, fille en jean fait un métier"

- "Qui me ressemble a sang bleu"

\section{LECTURES-ÉCRITURES CONCLUSIVES (ICI)}

Avant d'investir dans un travail de réécriture, plus ou moins distanciée de son modèle, leurs savoirs sociologiques et leurs expériences d'écritures les élèves, qui désormais maîtrisent mieux le réglage «idéologique» des textes, effectuent une lecture active et quasi "symptômale») des romans Harlequin.

Comme le recommandent les directives données par la maison d'édition à ses auteurs, les romans de la collection doivent nouer une intrigue amoureuse qui se dénoue par une "promesse de mariage à la dernière ligne du roman" (53) : mais les mariages "Harlequin" sont souvent le produit d'un véritable 《travail » qui leur permet d'accéder à la normalité. Prenons, à titre d'exemple, le roman de Violet Winspear intitulé "Pour un rêve d'amour" (1981) et observons comment s'opère le choix des partenaires et comment s'organisent leurs relations :

\section{a) choix des partenaires}

- de sexe opposé : dans un premier temps, seulement, l'héroïne vit dans un monde féminin (homosexualité latente ?) ; Rosary déclare ainsi à Lola, l'amie de son employeur: " Je ne suis pas en quête d'un mari. J'ai seulement l'intention d'accomplir au mieux ma tâche et de me faire aimer de Gisela. Je n'ai pas de... visées sur son père. - Peut-être changerez-vous d'avis (...) "). En effet quelques pages plus loin "rquelque chose la poussa à regarder de l'autre coté du patio (...). Avant même qu'il ne sorte de l'ombre, Rosary sut qu'il s'agissait de Dom Duarte de Montqueiro Ardo "s.

- unique : I'héroïne hésite un moment entre plusieurs hommes / Dacio était p/ein de charme et Rosary n'était pas femme à le nier ")/ pour finalement trouver l'homme de sa vie, un veuf qui va donc se remarier (polygamie latente).

- non incestueux : le couple Dom Duarte + Rosary est travaillé par la problématique incestueuse, même s'il est légalement normal: " Vous êtes si jeune (...). La jeunesse a besoin de la jeunesse (...). Vous n'étiez plus pour moi le professeur de ma fille... vous qui avez presque son âge" et qui êtes orpheline.

(51) Sur une approche sémiotique et structurale des proverbes, voir A.-J. Greimas, Du sens, Ed. du Seuil, 1970, pp. $309-314$.

(52) Sur La Table des Opérations Linguistiques et Littéraires Élémentaires, voir M. Benabou, in Protiques no 39 , p. 106.

(53) Cité par Y. Reuter, in Pratiques, no 32, p. 47 

évidente.

- matrimoniale : le mariage est vu comme une perspective relationnelle

- homogamique : cette règle est relativement transgressée (source probable de séduction, au moins passagère, sur un certain public); si l'amour l'emporte, c'est que l'hétérogamie est marquée comme superficielle, c'est-à-dire que la fiction nie implicitement les conséquences d'une telle alliance (54). Remarquons toutefois que, conformément aux tendances sociologiques analysées, si la jeune et séduisante professeur de piano, petite-fille d'un célèbre musicien, accède à la caste nobiliaire c'est au prix d'un (re)mariage avec un vieux beau.

- patriarcale : comme pour compenser le dérèglement homogamique, cette dimension est à la fois soulignée et acceptée, explicitement :

"Ma blonde esclave, j'avais le sentiment d'être un séducteur chaque fois que j'étais seul avec vous. - Et moi, j'avais l'impression d'être votre esclave chaque fois que vous posiez les yeux sur moi. - Je crois que vous n'appréciez guère ce genre d'impressions, declara-t-il sur un ton hésitant. - En vérité, fit-elle en levant sur lui un regard illuminé de bonheur, c'est l'impression la plus exaltante que j'aie jamais ressentie - vous êtes si jeune, chuchota-t-il en caressant ses cheveux. C'est injuste de... Mais d'un autre côté, ce serait inhumain de vous renvoyer en Angleterre, n'est-ce pas ma blonde esclave ? - Ce serait l'enfer ! - Préférez-vous rester? - Oui, ce serait le paradis - Alors entrons tous les deux au paradis!"'.

La lecture du roman, rose pervers, à la fois réactiverait la nostalgie de l'inceste et lui accorderait une satisfaction symbolique ; le lecteur serait saisi, un instant discursif, par " la douceur éternellement dénié à l'homme social, d'un monde où l'on pourrait vivre entre soi" (55). Les romans Harlequin présentent d'autres intérêts majeurs pour des lectures-écritures: leur aspect stéréotypé par exemple, dont on leur fait grief, fréquemment, au nom d'une esthétique de l'originalité et en fonction d'une consommation culturelle distinguee, est au contraire très précieux dans la perspective d'apprentissages scripturaux. Il est en effet possible, au lieu d'écraser les élèves sous la complexité sémiotique de textes hyper-élaborés et de paralyser leurs compétences en confrontant leurs productions aux Fuvres hyper-légitimées, de recenser sans difficulté par exemple les constantes syntaxiques et lexicales qui participent à la construction du portrait des héros/héroïnes ou des gestes amoureux/haineux des principaux personnages ; c'est la stéréotypie même de ces traits d'un roman à l'autre (chaque élève en lit au moins deux) qui permet de les lister rapidement et de pointer l'idéographie de la fiction (absence de séducteur noir dans les innombrables scènes américaines, maîtrise manifeste et systématique laissée à l'homme dans le rituel de courtoisie, etc...). Déplacer, recomposer ce puzzle narratif, y ajouter d'autres pieces devient alors pour l'apprenti scripteur un jeu... orienté.

Les élèves disposent encore, directement, des consignes d'écriture " maison» (décor, personnages, intrigue, composition, style, scènes à faire) qui «règlent » les superstructures textuelles et le niveau discursif proprement dit.

(54) F. Gretillat et alii, Une relation sans échange, rituels du couple dans un genre de littérature populaire, in Revue suisse de sociologie, 7, 1981, pp. 1-24.

(55) $\mathrm{Cl}$. Levi-Strauss, Les structures elémentaires de la parenté, Mouton, 1967. p. 570. 
La structure actancielle, très lisible dans ce type de récits, leur sert enfin de point de repère sûr dans la pratique scripturale. La consigne générale est la suivante : écrire, individuellement ou en groupes, un micro-roman (16 pages, le $1 / 10^{e}$ d'un Harlequin standard) destiné à paraître dans une plaquette collective distribuée sur le quartier; il s'agit de prendre une distance critique par rapport à trois consignes («maison »), disposition qui ménage pour chacun la liberté de se dégager plus ou moins du modèle de référence (un simple changement de décor n'a évidemment pas les mêmes conséquences qu'une permutation dans les rôles (56). Même si au cours de l'année certains textes classiques ont pu être («malmenés), il serait ruineux de forcer à la parodie. Selon leur sexe et leur trajectoire socio-scolaire les élèves n'ont en effet pas le même rapport au ("roman rose"): cette constatation est l'objet d'un éclaircissement et non prétexte à imposition d'une valeur, sur fond d'ethnocentrisme culturel. Voici, pour conclure, quelques extraits du récit d'un couple (!) d'élèves qui n'a eu garde d'oublier ni l'art du sous-entendu, ni celui du dialogue loufoque ou de l'interpellation brutale, tout en jouant, encore une fois, de l'onomastique, pour établir des relations matrimoniales troubles jusqu'à la perversion entre les un(e)s et les autres.

Et c'est bien parce que des règles d'écriture ont été explicitées et travaillées, une lecture sociologique organisée et structurée, que l'écriture d'un dé-règlement leur fut à la fois possible et peut-être... désirée! C. Eliane.

"T'as intérêt à être calé : on a été chienne avec toi» Fable. C. Corinne et

"Gloria, ravissante petite chienne au poil fauve qui lui tombait en cascade sur le bas des pattes, attendait désespérément un maitre. Gloria entra dans le chenil; ses beaux cheveux chatains, d'aspect soyeux, lui couvraient les épaules. Ses yeux verts parcouraient les cages lorsque son regard se fixa sur la merveilleuse Gloria. Après avoir fait le nécessaire pour vivre désormais avec cette adorable créature, Gloria ne put étouffer un petit cri quand Gloria accourut amoureusement vers elle.

Au même instant, Oscar ayant appris le décès de sa grand-tante se rendait chez le notaire pour la lecture du testament (...). En entrant dans la somptueuse demeure de la défunte (la plus belle de l'île), il constata que le notaire l'y attendait déjà. Dans la chambre, au-dessus du lit où était étendue la morte, il remarqua immédiatement un tableau le représentant aux côtés d'Oscar. Ce portrait était très réussi : on retrouvait bien le beau visage du jeune homme, avec ses yeux bleus-gris et ses cheveux bruns. I/ y figurait avec le beau colley au long pelage clair, car ils étaient les deux êtres que sa tante chérissait le plus au monde. La vue de ce tableau déplaisait fortement à Oscar car il détestait Oscar qui lui non plus ne le portait pas dans son cœur. I/ alla s'asseoir de façon à ne voir ni la peinture ni Oscar (lecteur, commence pas à japper, c'est que lé début). La sonnerie retentit et le notaire se précipita à la porte. Gloria et Gloria entrèrent alors dans la demeure de la feue vieille dame (...). Le notaire commença la lecture du testament de sa voix rauque (...). " C'est à mon petit neveu Oscar que je lègue mon bien le plus cher car je suis certaine qu'il en prendra soin: mon beau colley Oscar, le plus fidèle des compagnons (...) "). (t'as pas encore vu comme on a été chienne, lecteur).

Oscar, mal remis des émotions de la veille (et de la vieille), errait dans les rues de la ville. Absorbé par ses pensées, il mit un certain temps à reconnaitre Gloria, qu'il venait de croiser, en compagnie de Gloria. Comme la première fois, il remarqua la beauté et la gráce exceptionnelles - le chien quoi! - qui émanait de

(56) Sur les différences d'écriture de la parodie, voir A. Petitjean, Pratiques, no 42, pp. $63-78$. 
cette créature...(...). I/ venait de revoir Gloria, et déjà il ne pensait plus qu'à elle. Honteux de ce qui s'était passé, il rentra chez lui, "la queue entre les pattes "... (...) laïe ! ça se complique : lecteur, tu seras bientôt comme un chien dans un jeu de quilles).

- "Vous devez être heureux de vivre désormais sous le même toit qu'Oscar? Au fait pourquoi ne vous a-t-il pas accompagné, Gloria aurait pu discuter avec lui ? - Justement, le problème est là, nous nous entendons comme chien et chat. Je ne supporte pas cette vie constamment faite de disputes. Actuellement il ne sait pas où je suis; je suis parti sans l'avertir, et réciproquement - Vous n'exagérez pas un peu? - Non, malheureusement, j'ai un mal de chien à m'habituer à sa présence, dit-il en soupirant profondément - C'est étrange, il ne m'a pas paru désagréable - La question n'est pas là, son odeur ne m'est pas familière ; malgré tous mes efforts, je n'arrive pas à m'habituer (...). Lorsqu'il fut dans la rue, il se mit en quête d'un abri pour la nuit, mais n'étant pas habitué à coucher à la belle étoile, il se décida à rentrer (avec une certaine réticence) chez son maître. Oscar qui s'était cru débarrassé d'Oscar ne put retenir un énorme soupir en le voyant pénétrer dans le petit salon. Sans le savoir ils pensaient au même instant à la même chose: "Pourquoi la vie est-elle faite de contraintes? Comme si j'avais envie de vivre avec ce pouilleux!") (...). Tous deux se demandaient s'ils reverraient Gloria et Gloria. (Maintenant, accroche-toi, lecteur : tu vas avoir un mal de chien pour t'en sortir).

Le jour suivant, Oscar se réveilla le premier, vers neuf heures. I/ déjeuna copieusement. Oscar se leva à son tour et fit de même. Leurs esprits étaient toujours envahis par Gloria et Gloria. S'approchant de la fenêtre, Oscar n'en crut pas ses beaux yeux: elles passaient, toutes les deux! (...) On frappa à la porte. Son cœur se mit à battre, très vite. Il ouvrit et, à peine remis de ses émotions, resta immobile à admirer Gloria, plus ravissante que jamais. Oscar arriva à son tour et fit signe à Gloria d'entrer. Hésitante, elle regarda Gloria qui se décida à rompre le silence, remerciant Oscar de son invitation. "Entrez, je vous en prie... " articula difficilement Oscar après un court instant (...). Après un délicieux repas, ils poursuivaient une agréable conversation qu'Oscar coupa brièvement, en s'excusant de son mieux. II venait d'entendre un bruit sourd (...) et se dirigea vers le couloir accompagne d'Oscar (...). Oscar et Oscar se trouvèrent face à deux hommes armés, au masque bestial (...). - "Grand-tante Théodorine ne m'a laissé que cet animal (il désigna Oscar de la tête). - Tu t'fous d'moi ?..." (...) Gloria se leva d'un bond et se dirigea vers la porte afin de mieux comprendre la conversation. Gloria la suivait de très près (...) (N'aboie pas, lecteur, c'est pas fini!) (...). Le soir venu, Oscar et Oscar arrivèrent à l'heure juste. Gloria et Gloria, également ponctuelles, étaient prêtes (...). " Non, ce n'est pas possible, ils ne peuvent se reconnaître... La chienne, dans les bras d'un autre, I'homme masqué!" (...). Oscar songea: "Quelle vie de chien, quelle chienne de vie !" (...). I" régla l'addition et ils rentrèrent tous les deux, tristes comme deux chiens battus, qu'ils étaient (...). (Ne hurle pas à la mort, lecteur, ceci n'est qu'un livre) (...).

Grâce aux renseignements fournis par le flair d'Oscar, les ravisseurs (I'un d'eux avait amoureusement chloroforme Glorial furent identifiés par la police sous les pseudonymes de "Tristan le dur" et de "Husdent"; leurs portraitsrobots circulaient dans tous les journaux (...) (cette atmosphère de chien va bientot cesser, courage, lecteur!) (...).

Quand Gloria se réveilla, elle trouva Oscar couché près d'elle. "C'est vous Oscar!" s'exclama-t-elle. Folle de joie, elle voulait lui offrir ses lèvres et mille caresses; Oscar, qui bien sûr ignorait ses intentions, tourna instinctivement la tête : ils se rencontrèrent... Oscar et Gloria qui arrivaient en courant, cóte à côte, comme deux jeunes chiens fous, les trouvèrent ainsi, enlacés (...).

Tous quatre s'en allaient vers un futur improbable: /e temps venait de s'arrêter pour eux. I/s marchaient vers le bonheur et, passée la canicule, leurs silhouettes se dessinèrent une dernière fois dans le rouge du soleil couchant".. 